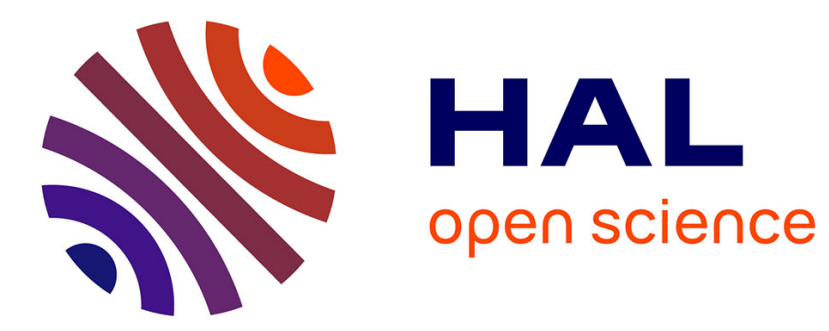

\title{
An integrated way to design FD/FTC modules via parity space and model following
}

Frédéric Hamelin, Taha Boukhobza, Hicham Jamouli, Cédric Join

\section{To cite this version:}

Frédéric Hamelin, Taha Boukhobza, Hicham Jamouli, Cédric Join. An integrated way to design FD/FTC modules via parity space and model following. Aug 2006, pp.CDROM. hal-00120921

\section{HAL Id: hal-00120921 \\ https://hal.science/hal-00120921}

Submitted on 18 Dec 2006

HAL is a multi-disciplinary open access archive for the deposit and dissemination of scientific research documents, whether they are published or not. The documents may come from teaching and research institutions in France or abroad, or from public or private research centers.
L'archive ouverte pluridisciplinaire HAL, est destinée au dépôt et à la diffusion de documents scientifiques de niveau recherche, publiés ou non, émanant des établissements d'enseignement et de recherche français ou étrangers, des laboratoires publics ou privés. 


\title{
AN INTEGRATED WAY TO DESIGN FD/FTC MODULES VIA PARITY SPACE AND MODEL FOLLOWING
}

\author{
F. Hamelin, T. Boukhobza, H. Jamouli and C. Join \\ Research Centre in Automatic Control (CRAN, CNRS UMR 7039), \\ Nancy-University, BP 239, 54506 Vandouvre, France, \\ email:frederic.hamelin@cran.uhp-nancy.fr
}

\begin{abstract}
This paper deals with the problem of integration of fault diagnosis and fault tolerant control modules. The main objective is to ensure a good behavior of the closedloop system in the presence of faults and disturbances. To this aim, a global active methodology is defined in order to synthesize an additive optimal control input from fault detection and isolation results. More precisely, a robust residual is firstly generated by means of usual parity relations to detect and isolate faults on the system. Next, a fault accommodation procedure, based model following control scheme, is used to generate an additive control input according to the residual characteristics. The efficiency of this methodology is illustrated through an heating system benchmark.
\end{abstract}

Keywords: Fault accommodation, parity-space approach, additive control law.

\section{INTRODUCTION}

In the past decades, there has been interest in synthesizing a FD/FTC system which has the ability to detect actuator/sensor faults automatically, and to maintain performances as close as possible to nominal ones after fault occurrence. Various approaches for FTC have been suggested in the literature, a survey overview is provided in Blanke et al. (2003), Hajiyev and Caliskan (2003) and Zhang and Jiang (2003).

FTC concepts can be classified into "active" and "passive" approaches. In the active approach (Noura et al. (2000), Wu et al. (2000), Siwakosit and Hess (2001), Zhang and Jiang (2001), Tao et al. (2002), Mahmoud et al. (2003)), a new set of control parameters is redesigned or selected such that the faulty system reaches the nominal system performance. Typically, the "active" approach consists in two parts:

- the diagnosis scheme;

- the controller reconfiguration.

In this approach, diagnosis and reconfiguration are thus strongly linked.
The passive approach makes use of reliable control techniques (Liang et al. (2000), Hsieh (2002), Ferreira (2002), Liao et al. (2002)) or robust control techniques (Zhou and Ren (2001), Niksefat and Sepehri (2002)) to ensure that the closed-loop system remains insensitive to certain faults.

Nearer to the first approach, a global approach integrating fault diagnosis and FTC is proposed in this paper. The main significance of this strategy consists in using a unique tool to diagnose and accommodate faults at once. It can be put in parallel with works of Zhang and Jiang (2001) which aim at using multiple model for control and fault detection. The proposed FTCS is based on the generation of parity relations (Chow and Willsky (1984)), which are useful to diagnose the system. By means of the residual which is issued from these relations, an additive control law is built to overcome the fault effect on the system. In a theoretical point of view, this FTCS is related to (Noura et al. (2000)) with an integration of fault detection results and predictive control tools.

The paper is organized as follows. The first part presents a general description of the fault 
accommodation problem based on the generation of an additive control law. Section 3 displays the theory used to compute this additive signal in the closed-loop framework. Stability and performance of the unknown input accommodation strategy are analyzed in the two next paragraphs. In section 6 , the proposed method is applied to an heating system benchmark. Finally, conclusions and prospects are included in section 7.

\section{PROBLEM STATEMENT}

Let us consider a discrete-time model of the nominal plant whose dynamics can be described by the following equations:

$$
\left\{\begin{aligned}
\underline{x}(k+1) & =A \underline{x}(k)+B \underline{u}(k)+E \underline{d}(k) \\
\underline{y}(k) & =C \underline{x}(k)+F \underline{d}(k)
\end{aligned}\right.
$$

where $\underline{x}(k) \in \mathbb{R}^{n}$ is the state vector, $y(k) \in \mathbb{R}^{p}$ is the output measurement vector, $\underline{u}(k) \in \mathbb{R}^{m}$ is the control vector. The components of vector $\underline{d}(k) \in \mathbb{R}^{n_{d}}$, which represent input disturbances, are all bounded and $A, B, C, E, F$ are constant and assumed to be known precisely with appropriate dimensions. In the following, all the matrices of (1) are assumed to be fixed and cannot be altered by the designer to improve performance.

In the presence of some additive sensor or actuator faults, the mathematical model of the LTI system (1) is given by:

$$
\left\{\begin{aligned}
\underline{x}(k+1) & =A \underline{x}(k)+B \underline{u}(k)+E \underline{d}(k)+G \underline{f}(k) \\
\underline{y}(k) & =C \underline{x}(k)+F \underline{d}(k)+H \underline{f}(k)
\end{aligned}\right.
$$

where $\underline{f}(k) \in \mathbb{R}^{n_{f}}$ denotes the fault vector and matrices $G, H$ are identified matrices, which characterize the distribution of failures acting directly on the dynamics (system and actuator faults) and on the measurements (sensor faults) respectively.

Stemming from the controller, $\underline{u}(k)$ is able to accommodate faults in the closed-loop framework if the feedback control system is designed to provide insensitivity to fault vector $f(k)$. However, in most cases, only limited faults can be rejected with conventional feedback control law. Indeed, increasing robustness to certain faults is only possible at the expense of decreased nominal performance. In order to reduce to zero the effects of particular faults, a control law reconfiguration system is often required.

In this paper, the problem consists in determining the control input $\underline{u}(k)$ such that real output $y(k)$ of the system tracks a desired reference trajectory $\underline{y}^{*}(k)$ when the system is affected by external faults $\underline{f}(k)$. Consequently, reconfigured control input $\underline{u}(k)$ is synthesized according to the definition of a "matched" system and aims at attenuating the variations in output $y(k)$ faced with unknown input $f(k)$. A representative block diagram of such a technique is shown in Figure 1.

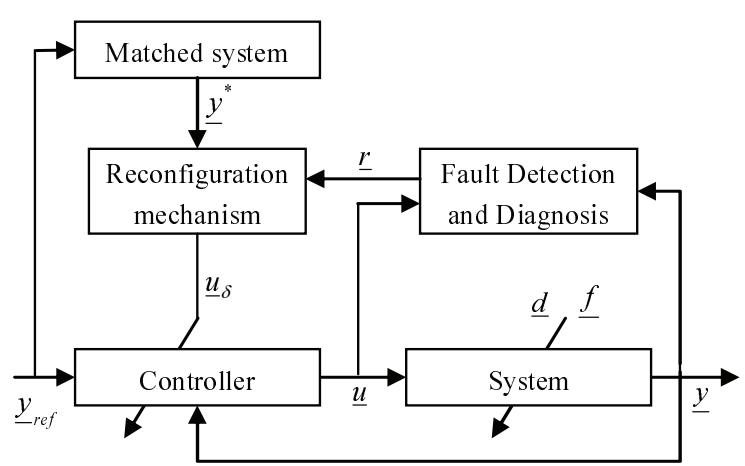

Fig. 1. Closed-loop and matched systems.

In fault-free case, it is important to point out that control law $\underline{u}(k)$ can be synthesized by any analytical method of controller design using output or state feedback. Next, for the sake of convenience, it is assumed that the general form of $\underline{u}(k)$ is given by the following output feedback:

$$
\begin{aligned}
\underline{u}(k) & =K_{p}\left(\underline{y}_{r e f}(k)-\underline{y}(k)\right) \\
& +K_{i} \sum_{j=0}^{k}\left(\underline{y}_{r e f}(k-j)-\underline{y}(k-j)\right)
\end{aligned}
$$

Controller matrices $K_{p}$ and $K_{i}$ are selected such that the closed-loop poles appear at the desired locations and the control system behaves as desired to set point changes $\underline{y}_{r e f}(k)$.

In faulty case, an additive control signal $\underline{u}_{\delta}(k)$ is synthesized. It tends to reduce the fault effects to zero and can be also a means of ensuring that the dynamic performance remains acceptable when a fault occurs. The dynamic response of the faulty closed-loop system is characterized by the reference model. The latter has been arranged to be forced with the same reference signal $\underline{y}_{r e f}(k)$ as that driving the basic closed-loop system. Consequently, the proposed FTCS is governed by only additive control $\underline{u}_{\delta}(k)$ and not by matrices $K_{p}$ and $K_{i}$, which are assumed to be identical to the nominal case. It is important to note that this assumption does not lead to a loss of generality.

The next section is dedicated to the optimal generation of additive control input $\underline{u}_{\delta}(k)$ in a closed-loop framework.

\section{OPTIMAL GENERATION OF ADDITIVE CONTROL INPUT IN FAULTY CASE}

In this section, a systematic approach is presented to generate optimal input $\underline{u}_{\delta}^{*}(k)$ in faulty case (the superscript $" * "$ denotes the optimal solution). Design objectives consist in defining $\underline{u}_{\delta}^{*}(k)$ such that predicted output $\hat{y}(k+i)=C \underline{\hat{x}}(k+i)$ of the process is close to reference trajectory $y^{*}(k+i)$ with $i=[1 \ldots s]$. Mathematically, $\underline{u}_{\delta}^{*}(k)$ is so the solution of the following optimization problem:

$$
\underline{u}_{\delta}^{*}(k)=\arg \min _{\underline{u}_{\delta}(k), \ldots, \underline{u}_{\delta}(k+s-1)} J
$$


with:

$$
J=\sum_{i=1}^{s}\left(\underline{\hat{y}}(k+i)-\underline{y}^{*}(k+i)\right)^{T} W_{i} W_{i}^{T}\left(\underline{\hat{y}}(k+i)-\underline{y}^{*}(k+i)\right)
$$

and $W_{i} \in \mathbb{R}^{p \times p}$ a weighting matrix.

To solve this problem, it is assumed that the general form of reconfigured control law $\underline{u}(k)$ using output feedback is given by:

$$
\begin{aligned}
\underline{u}(k) & =\underline{u}_{\delta}(k)+K_{p}\left(\underline{y}_{r e f}(k)-\underline{y}(k)\right) \\
& +K_{i} \sum_{j=0}^{k}\left(\underline{y}_{r e f}(k-j)-\underline{y}(k-j)\right)
\end{aligned}
$$

From equation (5), it appears that the control law input depends on the past samples $y(k-j)$. A clever technique can be used to adjust $\underline{u}(\bar{k})$ in a linear form of an augmented state vector. Indeed, if the latter is defined by $\underline{w}(k)=\left[\begin{array}{l}\underline{x}(k) \\ \underline{z}(k)\end{array}\right]$, with $\underline{z}(k)$ the integral of the output error, then the augmented state space equation is given by (6) according to (2) and (5):

$$
\begin{aligned}
\underline{w}(k+1)= & A_{c l} \underline{w}(k)+E_{c l} \underline{d}(k)+G_{c l} \underline{f}(k) \\
& +L_{c l} \underline{y}_{r e f}(k)+B_{c l} \underline{u}(k)
\end{aligned}
$$

with $A_{c l}=\left[\begin{array}{cc}A & 0 \\ -K_{i} C & I\end{array}\right], E_{c l}=\left[\begin{array}{c}E \\ -K_{i} F\end{array}\right], G_{c l}=$ $\left[\begin{array}{c}G \\ -K_{i} H\end{array}\right], L_{c l}=\left[\begin{array}{c}0 \\ K_{i}\end{array}\right]$ and $B_{c l}=\left[\begin{array}{c}B \\ 0\end{array}\right]$.

It follows that control input $\underline{u}(k)(5)$ becomes:

$$
\begin{aligned}
\underline{u}(k)=\underline{u}_{\delta} & (k)+\left(K_{p}+K_{i}\right) \underline{y}_{r e f}(k) \\
& -\left[\left(K_{p}+K_{i}\right) C-I\right] \underline{w}(k) \\
& -\left(K_{p}+K_{i}\right)(F \underline{d}(k)+H \underline{f}(k))
\end{aligned}
$$

If estimation $\hat{y}(k)=C \underline{x}(k)$ is expressed at times $k+1, \ldots, k \mp s$, the following matrix notation can be used:

$$
\begin{aligned}
& \underline{\hat{Y}}_{k+1, k+s}=M_{A_{c l}, C_{c l} \underline{w}}(k) \\
& +M_{A_{c l}, E_{c l}, C_{c l}, 0} \underline{\hat{D}}_{k, k+s}+M_{A_{c l}, G_{c l}, C_{c l}, 0} \underline{\hat{F}}_{k, k+s} \\
& +M_{A_{c l}, B_{c l}, C_{c l}, 0} \underline{U}_{k, k+s}+M_{A_{c l}, L_{c l}, C_{c l}, 0 \underline{Y}_{r e f, k, k+s}^{*}}
\end{aligned}
$$

where $C_{c l}=\left[\begin{array}{ll}C & 0\end{array}\right], \underline{\hat{D}}_{k, k+s}=\left[\begin{array}{c}\underline{\hat{d}}(k) \\ \vdots \\ \hat{d}(k+s)\end{array}\right]$ and $\underline{\hat{F}}_{k, k+s}$,

$\underline{U}_{k, k+s}, \underline{Y}_{r e f, k, k+s}^{*}, \underline{\hat{Y}}_{k+1, k+s}=C \underline{\hat{x}}_{k+1, k+s}$ are defined in the same manner as $\underline{\underline{D}}_{k, k+s}$.

Matrix $M_{A_{c l}, C_{c l}}$ is the extended observability matrix $\left[\begin{array}{c}C_{c l} A_{c l} \\ \vdots \\ C_{c l} A_{c l}^{s}\end{array}\right] \cdot M_{N, P, Q, R}$ is of Toeplitz form and is built up of the matrix elements $N, P, Q, R$ :

$$
M_{N, P, Q, R}=\left[\begin{array}{ccccc}
Q P & R & 0 & \cdots & 0 \\
Q N P & Q P & R & \ddots & \vdots \\
\vdots & \ddots & \ddots & \ddots & 0 \\
Q N^{s-1} P & \cdots & Q N P & Q P & R
\end{array}\right]
$$

For the sake of convenience, the desired reference trajectory $\underline{y}^{*}(k)$, which is used to control the faulty process, is chosen similar to $C_{c l} \underline{w}^{*}(k)$, where $\underline{w}^{*}(k)$ is defined by the following state-space representation:

$$
\begin{aligned}
\underline{w}^{*}(k+1)= & A_{c l} \underline{w}^{*}(k)+L_{c l} \underline{y}_{r e f}(k) \\
& +B_{c l}\left(\underline{u}(k)-\underline{u}_{\delta}(k)\right)
\end{aligned}
$$

Collecting the next i-step reference outputs for $i=1, \ldots, s$ in a matrix notation similar to (8) yields:

$$
\begin{aligned}
& \underline{Y}_{k+1, k+s}^{*}=M_{A_{c l}, C_{c l}} \underline{w}^{*}(k) \\
& \quad+M_{A_{c l}, L_{c l}, C_{c l}, 0} \underline{Y}_{r e f, k, k+s}^{*} \\
& \quad+M_{A_{c l}, B_{c l}, C_{c l}, 0}\left(\underline{U}_{k, k+s}-\underline{U}_{\delta, k, k+s}\right)
\end{aligned}
$$

Substituting Eq (8) and (10) in criterion (4), it can easily be shown that $\underline{U}_{\delta, k, k+s}^{*}$ (where the superscript $" *$ " denotes the optimal solution) can be calculated minimizing the equivalent criterion function:

$$
\begin{aligned}
& J=\| W^{T}\left(M_{A_{c l}, C_{c l}}\left(\underline{w}(k)-\underline{w}^{*}(k)\right)\right. \\
& +M_{A_{c l}, B_{c l}, C_{c l}, 0} \underline{U}_{\delta, k, k+s}+M_{A_{c l}, E_{c l}, C_{c l}, 0} \underline{\hat{D}}_{k, k+s}
\end{aligned}
$$

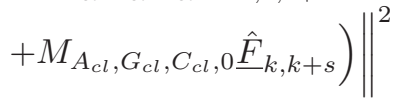

where $W=\operatorname{diag}\left(W_{i}\right)$ is a constraint matrix of dimension $(s . p) \times(s . p)$ collecting the $s$ elementary matrices $W_{i}$.

Clearly, a problem occurs when solving $\underline{U}_{\delta, k, k+s}$ since it involves the knowledge of vectors $\underline{D}_{k, k+s}$ and $\underline{\hat{F}}_{k, k+s}$. However, according to (Chow and Willsky (1984)) and from the faulty system model (6), analytical redundancy relations can be used to approximate criterion $J$ and to generate a residual $\underline{r}(k)$, useful for fault detection and isolation:

$$
\begin{aligned}
& \quad \underline{r}(k)=V^{T}\left(\underline{Y}_{k-s+1, k}\right. \\
& \left.-M_{A_{c l}, L_{c l}, C_{c l}, 0} \underline{Y}_{r e f, k-s, k}^{*}-M_{A_{c l}, B_{c l}, C_{c l}, 0} \underline{U}_{k-s, k}\right) \\
& =V^{T}\left(M_{A_{c l}, C_{c l}} \underline{w}(k-s)\right. \\
& \left.+M_{A_{c l}, E_{c l}, C_{c l}, F} \underline{D}_{k-s, k}+M_{A_{c l}, G_{c l}, C_{c l}, H} \underline{F}_{k-s, k}\right)
\end{aligned}
$$

Indeed, if it is assumed that no information is available concerning the next $s$ samples of $\underline{d}(k)$ and $\underline{f}(k)$ (from $t=k$ to $t=k+s$ ), a simple approximation consists in putting back terms $\underline{D}_{k-s, k}$ and $\underline{F}_{k-s, k}$ in the place of $\underline{\hat{D}}_{k, k+s}$ and $\underline{\hat{F}}_{k, k+s}$ respectively. This approximation is equivalent to assuming that there are no "severe" changes in the nature of the disturbances and the faults over the next $s$ samples. It is important to point out that there is sense in doing this approximation for $f(k)$ since optimal control law $\underline{u}_{\delta}^{*}(k)$ is useful only after fault occurrence. Indeed, let us remind that the synthesis procedure of $\underline{u}_{\delta}^{*}(k)$, which is integrated in an active FTCS, is added to the nominal one $\underline{u}(k)$ (as mentioned in (5)) only when a fault is detected by the FDI system. Although the last

term of (12) involves the unknown $\underline{w}(k-s), \underline{D}_{k-s, k}$ and $\underline{F}_{k-s, k}$, note that residual $\underline{r}(k)$ can be calculated by means of (12) using outputs $\underline{Y}_{k-s+1, k}$ and inputs $\underline{U}_{k-s, k}, \underline{Y}_{r e f, k-s, k}^{*}$ available at $t=k$. So, if it is assumed that the full row rank matrix $V^{T} \in \mathbb{R}^{(q \times s . p)}$ 
is related to the full row rank matrix $V_{1}^{T}$ by relation $V^{T}=W^{T} V_{1}\left(V_{1}^{T} V_{1}\right)^{-1} V_{1}^{T}$ in order to make insensitive residual $\underline{r}(k)$ to measurement disturbances and sensor faults (note that $\underline{r}(k)$ remains sensitive to state disturbances and actuator/system faults):

$$
\left\{\begin{array}{l}
V_{1}^{T} M_{A_{c l}, E_{c l}, C_{c l}, F}=V_{1}^{T} M_{A_{c l}, E_{c l}, C_{c l}, 0} \\
V_{1}^{T} M_{A_{c l}, G_{c l}, C_{c l}, H}=V_{1}^{T} M_{A_{c l}, G_{c l}, C_{c l}, 0}
\end{array}\right.
$$

then residual $r(k)$ can be changed into:

$$
\begin{gathered}
\underline{r}(k)=V^{T}\left(\underline{Y}_{k-s+1, k}\right. \\
\left.-M_{A_{c l}, L_{c l}, C_{c l}, 0} \underline{Y}_{r e f, k-s, k}^{*}-M_{A_{c l}, B_{c l}, C_{c l}, 0} \underline{U}_{k-s, k}\right) \\
=V^{T}\left(M_{A_{c l}, C_{c l}} \underline{w}(k-s)\right. \\
\left.+M_{A_{c l}, E_{c l}, C_{c l}, 0} \underline{D}_{k-s, k}+M_{A_{c l}, G_{c l}, C_{c l}, 0} \underline{F}_{k-s, k}\right)
\end{gathered}
$$

and criterion $J$ can be replaced by $J_{1}$, which does not require the knowledge of unknown inputs and faults:

$$
\begin{aligned}
& J_{1}=\| \underline{r}(k)+V^{T}\left(M_{A_{c l}, C_{c l}}\left(\underline{w}(k)-\underline{w}^{*}(k)\right)\right. \\
& \left.-M_{A_{c l}, C_{c l}} \underline{w}(k-s)+M_{A_{c l}, B_{c l}, C_{c l}, 0} \underline{U}_{\delta, k, k+s}\right) \|^{2}
\end{aligned}
$$

Since $J_{1}$ depends on the residual $\underline{r}(k)$, it has to be optimized on line in order to synthesize the incremental control input $\underline{u}_{\delta}^{*}(k)$. Consequently, at time $t=k$, only additive control input $\underline{u}_{\delta}(k)$ is useful among all the components of $\underline{U}_{\delta, k, k+s}$. Future samples $\underline{u}_{\delta}(k+j)$ (with $j>0$ ) are interesting to compute only at samples $t=k+j$. According to the structure of $\underline{U}_{\delta, k, k+s}$, criterion function (15) is then rewritten:

$$
\begin{aligned}
& J_{1}=\| \underline{r}(k)+V^{T}\left(M_{A_{c l}, C_{c l}}\left(\underline{w}(k)-\underline{w}^{*}(k)\right)\right. \\
& -M_{A_{c l}, C_{c l}} \underline{w}(k-s)+P_{\left.A_{c l}, B_{c l}, C_{c l}, 0 \underline{u}_{\delta}(k)\right)} \|^{2}
\end{aligned}
$$

where $P_{A_{c l}, B_{c l}, C_{c l}, 0}=M_{A_{c l}, B_{c l}, C_{c l}, 0}\left[\begin{array}{lll}I_{m} & \cdots & I_{m}\end{array}\right]^{T}$ allows us to simulate a constant incremental control input from $t=k$ to $t=k+s$.

If $\left\|V^{T} P_{A_{c l}, B_{c l}, C_{c l}, 0}\right\| \neq 0$, a global minimum of $J_{1}$ respect to $\underline{u}_{\delta}(k)$ can be obtained by setting the gradient equal to zero:

$$
\begin{gathered}
\underline{u}_{\delta}^{*}(k)=\left(V^{T} P_{A_{c l}, B_{c l}, C_{c l}, 0}\right)^{+}(-\underline{r}(k) \\
\left.+V^{T}\left(M_{A_{c l}, C_{c l}}\left(\underline{w}^{*}(k)-\underline{w}(k)\right)+M_{A_{c l}, C_{c l}} \underline{w}(k-s)\right)\right)
\end{gathered}
$$

In order to limit the complexity and the computation time of $\underline{u}_{\delta}^{*}(k)$, another constraint can be imposed on the definition of $V$. One way to avoid the estimation of the state vector $\underline{w}(k)$ at times $k$ and $k-s$ is by choosing $V^{T}=W^{T} V_{1}\left(V_{1}^{T} V_{1}\right)^{-1} V_{1}^{T}$ such that the term $\left(M_{A_{c l}, C_{c l}}\left(\underline{w}^{*}(k)-\underline{w}(k)\right)+M_{A_{c l}, C_{c l}} \underline{w}(k-s)\right)$ is eliminated in the definition of $\underline{u}_{\delta}^{*}(k)$ :

$$
V_{1}^{T} M_{A_{c l}, C_{c l}}=0
$$

Taking this equality constraint into account, the input $\underline{u}_{\delta}^{*}(k)$ is finally calculated by means of the following relation:

$$
\underline{u}_{\delta}^{*}(k)=-\left(V^{T} P_{A_{c l}, B_{c l}, C_{c l}, 0}\right)^{+} \underline{r}(k)
$$

Note that the space spanned by the set of the q columns of $V(18)$ is commonly used for fault detection and isolation and is called the parity space (Chow and Willsky (1984)). In practical terms, this integrated FD/FTC approach can be represented by the block diagram in Figure 2.

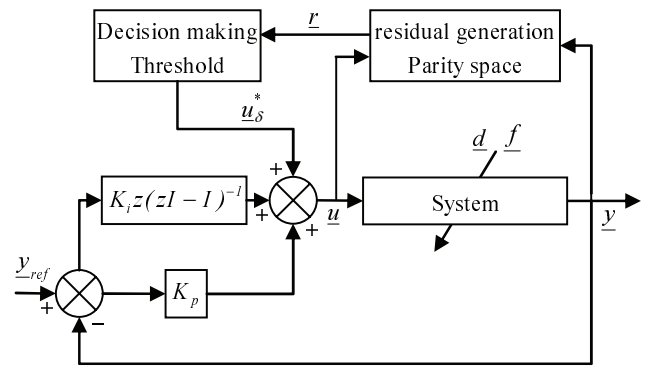

Fig. 2. Closed-loop system driven by $\underline{u}_{\delta}^{*}$ and $\underline{y}_{r e f}$.

Next, necessary and sufficient conditions are derived which ensure the stability of the closed-loop system when they are satisfied. Indeed, it is important to investigate the influence of the incremental signal $\underline{u}_{\delta}(k)$ on the properties of the closed-loop system.

\section{STABILITY OF THE FAULTY CLOSED-LOOP SYSTEM}

To check whether the closed-loop system is stable, it is necessary to verify that all its poles lie inside the unit circle. Assuming that $\underline{f}(k)$ and $\underline{d}(k)$ are not function of the state and substituting (7) into (6), we see that the state space vector $\underline{w}(k)$ can be expressed as a linear function of inputs $\underline{d}(k), \underline{f}(k), \underline{y}_{r e f}(k)$ and $\underline{u}_{\delta}(k)$, that is,

$$
\begin{aligned}
\underline{w}(k+1)= & A_{c l}^{*} \underline{w}(k)+E_{c l}^{*} \underline{d}(k)+G_{c l}^{*} \underline{f}(k) \\
& +L_{c l}^{*} \underline{y}_{r e f}(k)+B_{c l}^{*} \underline{u}_{\delta}(k)
\end{aligned}
$$

where $\quad A_{c l}^{*} \quad=\left[\begin{array}{cc}A-B\left(K_{p}+K_{i}\right) C & B \\ -K_{i} C & I\end{array}\right]$, $B_{c l}^{*}=\left[\begin{array}{c}B \\ 0\end{array}\right], \quad E_{c l}^{*}=\left[\begin{array}{c}E-B\left(K_{p}+K_{i}\right) F \\ -K_{i} F\end{array}\right]$, $G_{c l}^{*}=\left[\begin{array}{c}G-B\left(K_{p}+K_{i}\right) H \\ -K_{i} H\end{array}\right]$ and $L_{c l}^{*}=\left[\begin{array}{c}B\left(K_{p}+K_{i}\right) \\ K_{i}\end{array}\right]$.

Substitution of (19) into (20) yields for $\underline{w}(k)$ :

$$
\begin{aligned}
& \underline{w}(k+1)=A_{c l}^{*} \underline{w}(k)+E_{c l}^{*} \underline{d}(k)+G_{c l}^{*} \underline{f}(k) \\
& \quad+L_{c l}^{*} \underline{y}_{r e f}(k)-B_{c l}^{*}\left(V^{T} P_{A_{c l}, B_{c l}, C_{c l}, 0}\right)^{+} \underline{r}(k)
\end{aligned}
$$

Because (21) is derived under the assumption that $V^{T} M_{A_{c l}, C_{c l}}=0$, the residual $\underline{r}(k)$ is insensitive to $\underline{w}(k-s)(12)$ and the model dynamics are characterized by:

$$
\begin{gathered}
\underline{w}(k+1)=A_{c l}^{*} \underline{w}(k)+E_{c l}^{*} \underline{d}(k)+G_{c l}^{*} \underline{f}(k)+L_{c l}^{*} \underline{y}_{r e f}(k) \\
-B_{c l}^{*}\left(V^{T} P_{A_{c l}, B_{c l}, C_{c l}, 0}\right)^{+} V^{T} \\
\left(M_{A_{c l}, E_{c l}, C_{c l}, 0} \underline{D}_{k-s, k}+M_{A_{c l}, G_{c l}, C_{c l}, 0} \underline{F}_{k-s, k}\right)
\end{gathered}
$$

Note that the closed-loop system shown in block diagram form in Figure 2 is globally asymptotically stable whatever value of $V$ such that $V^{T} M_{A_{c l}, C_{c l}}=0$ if and only if $\left|\lambda_{i}\left(A_{c l}^{*}\right)\right|<1, i=1, \ldots, n$. 
So far we have discussed a stable unknown input accommodation procedure using $\underline{u}_{\delta}^{*}(k)$. In the following, the performance of the unknown input accommodation strategy is analyzed.

\section{FD/FTC PERFORMANCES OF THE ACTIVE FAULT-TOLERANT STRATEGY}

Using (14), (16) and (19), performance of the disturbance/fault accommodation system is evaluated by the criterion $J_{1}$ :

$$
\begin{aligned}
& J_{1}=\| V^{T}\left(I-P_{A_{c l}, B_{c l}, C_{c l}, 0}\left(V^{T} P_{A_{c l}, B_{c l}, C_{c l}, 0}\right)^{+} V^{T}\right) \\
& \left(M_{A_{c l}, E_{c l}, C_{c l}, 0} \underline{D}_{k-s, k}+M_{A_{c l}, G_{c l}, C_{c l}, 0} \underline{F}_{k-s, k}\right) \|^{2}
\end{aligned}
$$

The above-mentioned criterion can be regarded as an approximation of the sum of squares of the errors between the predicted output of the process $\underline{\hat{y}}(k)$ and the desired process output $\underline{y}^{*}(k)$ in the next $s$ samples. Clearly, the part played by the incremental signal $\underline{u}_{\delta}^{*}(k)$, which is reflected by the term $P_{A_{c l}, B_{c l}, C_{c l}, 0}\left(V^{T} P_{A_{c l}, B_{c l}, C_{c l}, 0}\right)^{+} V^{T}$ in (23), is to reduce the effects of $\underline{d}(k)$ and $f(k)$. However, according to the internal form of residual:

$$
\begin{aligned}
& \underline{r}(k)=V^{T} \\
& \left(M_{A_{c l}, E_{c l}, C_{c l}, 0} \underline{D}_{k-s, k}+M_{A_{c l}, G_{c l}, C_{c l}, 0} \underline{F}_{k-s, k}\right)
\end{aligned}
$$

it appears that $\underline{r}(k)$ can become insensitive to some disturbances or faults if the latter are not reflected by $\underline{D}_{k-s, k}$ and $\underline{F}_{k-s, k}$, but only through state vector $M_{A_{c l}, C_{c l}} \underline{w}(k-s)$. Hence, it is not judicious to model faults and/or disturbances by increasing the dimension of the state vector $\underline{w}(k)$ when $V$ is chosen orthogonal to $M_{A_{c l}, C_{c l}}$. Note that this conclusion is only true for fault and disturbance accommodation. It is indeed easier to detect and isolate faults when they are defined by particular deterministic functions. In FDI framework, $V^{T}=W^{T} V_{1}\left(V_{1}^{T} V_{1}\right)^{-1} V_{1}^{T}$ results from minimizing the performance index:

$$
\frac{\left\|V_{1}^{T} M_{A_{c l}, E_{c l}, C_{c l}, 0}\right\|^{2}}{\left\|V_{1}^{T} M_{A_{c l}, G_{c l}, C_{c l}, 0}\right\|^{2}}
$$

\section{APPLICATION}

To illustrate the effectiveness of the methodology, some tests have been carried out using the model of an heating system benchmark. Figure 3 shows the schematic diagram of the whole plant that is considered further.

Two tanks (1 and 2) are used for pre-heating the liquid flow, which is provided by two electrically powered pumps. The liquid temperatures are adjusted in these two tanks by means of two thermal resistances. A third tank discharges to atmospheric pressure and makes possible the mixing of the two liquids coming from the two-pre-heating tanks.

The general state space representation $\underline{\mathrm{x}}(\mathrm{k}+1)=$ $A \underline{x}(k)+B \underline{u}(k)$ can be used to describe the behavior

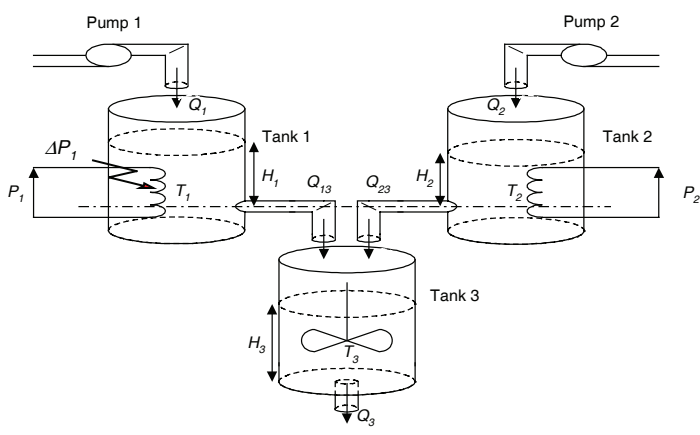

Fig. 3. Heating system benchmark.

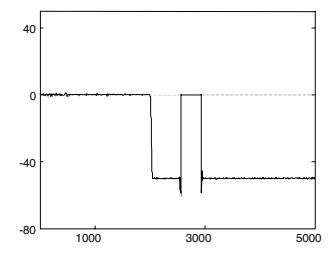

Fig. 4. Residual $r_{2}(k)$.

of this nonlinear system around the specific set-point: $H_{1,0}=40 \mathrm{~cm}, H_{2,0}=30 \mathrm{~cm}, H_{3,0}=20 \mathrm{~cm}, T_{1,0}=17^{0} \mathrm{C}$, $T_{2,0}=22^{0} \mathrm{C}, T_{3,0}=19.8^{0} \mathrm{C}, Q_{1,0}=13.33 \times 10^{-6} \mathrm{~m}^{3} \mathrm{~s}^{-1}$, $Q_{2,0}=16.67 \times 10^{-6} \mathrm{~m}^{3} \mathrm{~s}^{-1}, P_{1,0}=111.5 \mathrm{~W}$ and $P_{2,0}=139.3 \mathrm{~W}$. State-vector $\underline{x}(k)$ is such that $\underline{x}(k)=\left[h_{1}(k) h_{2}(k) h_{3}(k) t_{1}(k) t_{2}(k) t_{3}(k)\right]$ whereas control law is defined by $\underline{u}(k)=$ $\left[q_{1}(k) q_{2}(k) p_{1}(k) p_{2}(k)\right]$.

\subsection{Control aims and results}

The objectives of the system are to adjust the fluid level $H_{3}(k)$ and the fluid temperature $T_{3}(k)$ according to the reference variables $H_{3, \text { ref }}(k)$ and $T_{3, \text { ref }}(k)$. Controller matrices $K_{p}$ and $K_{i}$ are selected such that:

$$
K_{p}=\left[\begin{array}{cc}
10^{-4} & 10^{-6} \\
10^{-4} & 10^{-7} \\
500 & 50 \\
1000 & 250
\end{array}\right], K_{i}=\left[\begin{array}{cc}
2 \times 10^{-7} & -10^{-9} \\
2 \times 10^{-7} & -10^{-8} \\
0.7 & 0.4 \\
4 & 0.5
\end{array}\right]
$$

During time interval $[2000,2570]$ and from time 2930 seconds, a fault occurs on actuator $P_{2}$. It consists in a reduction of $50 \%$ of its gain $\left(f_{P_{2}}(k)=\right.$ $\left.0.5\left(P_{2,0}+p_{2}(k)\right)\right)$.

By means of residual $\underline{r}(k)$ (Figure 4$)$, which is used to define the incremental control input $\underline{u}_{\delta}^{*}(k)$, the fault on $P_{2}$ can be detected and estimated in per cent. Indeed, as $V$ is chosen to be orthogonal to the extended observability matrix $M_{A_{c l}, C_{c l}}$, residual $\underline{r}(k)$ is only sensitive to the fault occurring on actuator $P_{2}$ and disturbances. Consequently, if $V$ is defined as a vector belonging to the parity space and maximizing the sensitivity of the residual on the actuator fault, the loss of control effectiveness in $P_{2}$ can be estimated on-line (Figure 4 ) by means of residual $r_{2}(k)$ defined by:

$$
r_{2}(k)=\frac{\underline{r}(k)}{V^{T} M_{A_{c l}, G_{c l}, C_{c l}, 0}\left(P_{2,0}+p_{2}(k)\right)}
$$

For this example of application, the design parameters are defined with $s=5$ and $W=I$. 
On each of the figures (5) is presented the output $T_{3}(k)$ with two different values for $\underline{u}_{\delta}(k)$, which is used once the fault has been detected by $r_{2}(k)$.

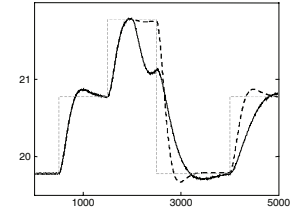

(a) $\underline{u}_{\delta}(k)=\underline{0}$

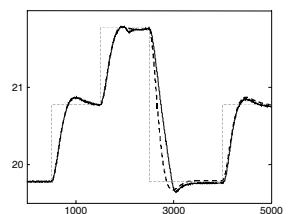

(b) $\underline{u}_{\delta}(k)=\underline{u}_{\delta}^{*}(k)$
Fig. 5. Measurement $T_{3}(k)$ with different values for $\underline{u}_{\delta}(k)$.

Desired reference trajectory $\underline{y}^{*}(k)$ is represented in broken line whereas $\underline{y}_{r e f}(k)$ is in dotted line. It can be seen from the figures that the incremental control law $\underline{u}_{\delta}^{*}(k)$ gives better tracking performance than $\underline{u}_{\delta}(k)=\underline{0}$ when the fault occurs. The time response of $H_{3}(k)$ is not given because it is not affected by the fault. On the other hand, it is interesting to compare the control inputs $\underline{u}(k)$ of the system with and without additional term $\underline{u}_{\delta}^{*}(k)$ (Figure 6 ).

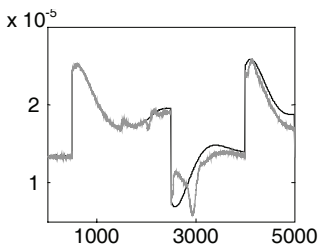

(a) $Q_{1}(k)$

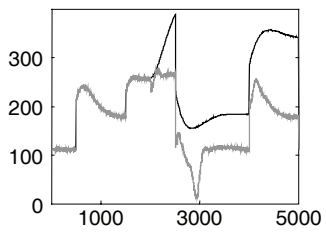

(c) $P_{1}(k)$

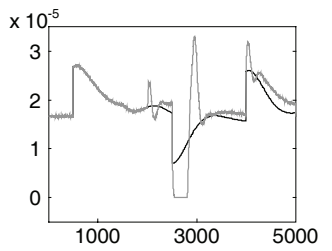

(b) $Q_{2}(k)$

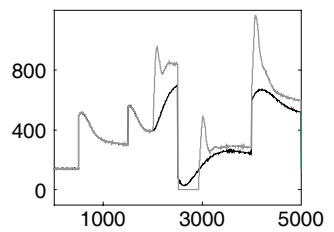

(d) $P_{2}(k)$
Fig. 6. Control input $\underline{u}(k)$ with $\underline{u}_{\delta}(k)=\underline{0}$ and $\underline{u}_{\delta}(k)=\underline{u}_{\delta}^{*}(k)$.

We note that the time response of $\underline{u}(k)$ is significantly changed when the fault occurs according to the effects of the additional control input $\underline{u}_{\delta}^{*}(k)$.

\section{CONCLUSION}

This paper has presented an integrated method for FDI and FTC based on the generation of a detection residual in the time domain. The properties of the traditional parity space approach and some concepts of predictive control have been used to synthesize an incremental control input. The stability of the FTC strategy has been studied. The proposed method has been applied to an heating system benchmark. Further research concerns the use of an augmented criterion function (4) establishing a compromise between the effort of the actuators and the tracking error. Furthermore, it would be interesting to study the sensitivity of this approach to model uncertainties.

\section{REFERENCES}

Blanke, M., M. Kinnaert, J. Lunze and M. Staroswiecki (2003). Diagnosis and fault-tolerant control, Springer Verlag, Heidelberg.

Chow, E.Y. and A.S. Willsky (1984). 'Analytical redundancy and the design of robust failure detection systems', IEEE Transactions on Automatic Control, AC-29(7), 603-614.

Ferreira, P. (2002). 'Tracking with sensor failures', Automatica, 38(9), 1621-1623.

Hajiyev, C. and F. Kaliskan (2003). Fault diagnosis and reconfiguration in flight control systems, Kluwer academic publishers, Boston.

Hsieh, C. (2002). 'Performance gain margins of the two-stage LQ reliable control', Automatica, 38(11), 1985-1990.

Liang, Y., D.C. Liaw and T.C. Lee (2000). 'Reliable control of non-linear systems', IEEE Transactions on Automatic Control, 45(4), 706-710.

Liao, F., J. Wang and G. Yang (2002). 'Reliable robust tracking control: an LMI approach', IEEE Transactions on Control Systems Technology, 10(1), 76-89.

Mahmoud, M., J. Jiang and Y. Zhang (2003). Active fault tolerant control systems: stochastis analysis and synthesis, Springer-Verlag, Berlin.

Niksefat, N. and N. Sepehri (2002). 'A QFT faulttolerant control for electrohydraulic positioning systems', IEEE Transactions on Control Systems Technology, 10(4), 626-632.

Noura, H., D. Sauter, F. Hamelin and D. Theilliol (2000). 'Fault-Tolerant control in dynamic system. Application to a winding machine', IEEE Control System Magazine, 20, 33-39.

Siwakosit, W. and R. Hess (2001). 'Multi-input/multioutput reconfigurable flight control design', Journal of Guidance, Control, and Dynamics, 24(6), 1079 1088.

Tao, G., S. Chen and S. Joshi (2002). 'An adaptive actuator failure compensation controller using output feedback', IEEE Transactions on Automatic Control, 47(3), 506-511.

Wu, N., Y. Zhang and K. Zhou (2000). 'Detection, estimation, and accommodation of loss of control effectiveness', International Journal of Adaptive Control and Signal Processing, 14, 775-795.

Zhang, Y. and J. Jiang (2001). 'Integrated active fault-tolerant control using IMM Approach', IEEE Transactions on Aerospace and Electronic Systems, 37(4).

Zhang, Y. and J. Jiang (2003). Bibliographical review on reconfigurable fault-tolerant control systems, 265-276, Proc. of IFAC Safeprocess, Washington, USA.

Zhao, Q. and J. Jiang (1998). 'Reliable state feedback control systems design against actuator failures', Automatica, 34(10), 1267-1272.

Zhou, K. and Z. Ren (2001). 'A new controller architecture for high performance, robust, and faulttolerant control', IEEE Transactions on Automatic Control, 46(10), 1613-1618. 